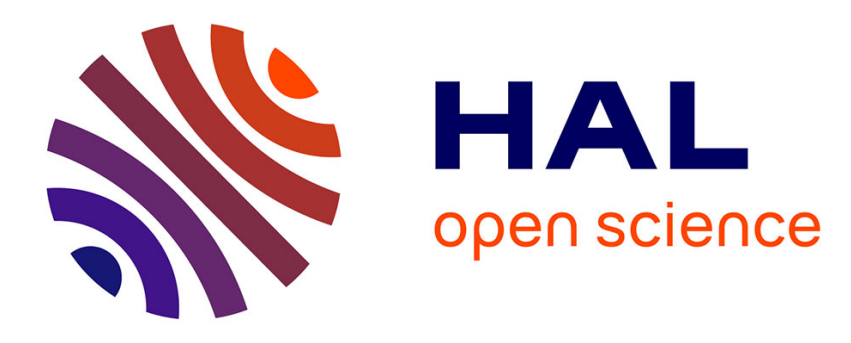

\title{
Analytical Solution for Joint Coupling in NAO Humanoid Hips
}

Vincent Hugel, Nicolas Jouandeau

\section{To cite this version:}

Vincent Hugel, Nicolas Jouandeau. Analytical Solution for Joint Coupling in NAO Humanoid Hips. 18th annual RoboCup International Symposium, Jul 2014, Joaõ Pessoa, Brazil. 10.1007/978-3-31918615-3_35. hal-02317150

\section{HAL Id: hal-02317150 \\ https://hal.science/hal-02317150}

Submitted on 15 Oct 2019

HAL is a multi-disciplinary open access archive for the deposit and dissemination of scientific research documents, whether they are published or not. The documents may come from teaching and research institutions in France or abroad, or from public or private research centers.
L'archive ouverte pluridisciplinaire HAL, est destinée au dépôt et à la diffusion de documents scientifiques de niveau recherche, publiés ou non, émanant des établissements d'enseignement et de recherche français ou étrangers, des laboratoires publics ou privés. 


\title{
Analytical Solution for Joint Coupling in NAO Humanoid Hips
}

\author{
Vincent Hugel $^{1}$ and Nicolas Jouandeau ${ }^{2}$ \\ 1 LISV, University of Versailles, France \\ 2 LIASD, University of Paris 8, France
}

\begin{abstract}
Usually the legs of humanoids capable of omnidirectional walking are not underactuated. In other words each one of the six degrees of freedom of the torso can be commanded independently from the leg joint angles. However the NAO humanoid robot has a coupled joint at the hips, which makes 11 degrees of freedom instead of 12 for the locomotor apparatus. As a consequence the trunk of the robot has only 5 independent degrees of freedom when the positions of both feet are fixed, and each leg cannot be commanded independently to execute walking steps. Up to now only bypass solutions have been proposed, where the coupled joint angles are not calculated exactly. This paper describes an analytical solution to determine the exact angle to be applied to the coupled joint. The method uses the positions of both foot ankles in the trunk reference frame and the angle between footprints as inputs, and calculates the yaw angle of the trunk. The solution was demonstrated in a dynamics simulator using the NAO model.
\end{abstract}

\section{Introduction}

The NAO humanoid robot [1] is widely used in the robotics community to design omnidirectional walking gaits $[2,3]$, kicking moves [4], and stabilizers [5,6]. In the NAO robot the yaw joints at the hips are coupled and $45[\mathrm{deg}]$ inclined with respect to the vertical [1] (Fig. 1). Therefore the hips of NAO are underactuated, with five independent degrees of freedom instead of six.
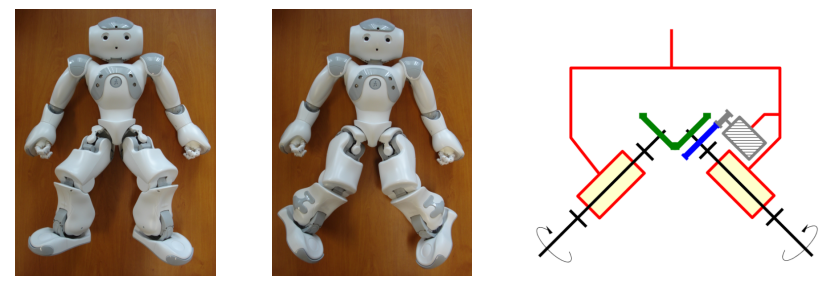

Fig. 1: Coupled hip-yaw rotary joints of the NAO pelvis. 
To deal with this underactuation, Graf et al. $[3,7]$ introduced a fictive yaw joint at the swing foot, and applied a 6-DOF Inverse Geometric Model (IGM) based on the kinematic chain of this virtual leg, the yaw-pitch joint at the hip being fixed by the positioning of the support leg. However non-zero angle values for the fictive yaw joint constitute positioning errors. Nonetheless such errors have less impact than errors that would result from the addition of a virtual pitch or a virtual roll joint. Alcaraz-Jiménez [8] defined an iterative process to evaluate the torso yaw angle by using a proportional law that tends to minimize the yaw angle error of the swing foot. The torso yaw angle is then used by the inverse kinematics to update the joint angles. The process is iterated three sampling times to limit the control delay. Hugel et al. [9] used the torso longitudinal axis as a symmetry axis. They considered that the yaw angle between this axis and the right foot is the same as the yaw angle between this axis and the left foot. The symmetry property is only valid when both feet are on the ground, and is limited to the turning steps whose center is located on the torso axis. This method is still an approximation when the leg is lift off in this kind of turning steps.

All the methods mentioned above are approximation methods since they do not calculate the coupled joint angle exactly. This paper proposes an analytical solution that calculates the yaw angle of the trunk exactly given the positions of both feet in the Trunk Coordinate Frame (TCF) and the horizontal angle between the feet. This allows calculating the orientation of each foot within the TCF before applying the IGM to get all joint angles to command the legs to achieve the desired motion.

Section 2 presents the modeling convention used for the geometric calculations. Section 3 describes the leg model similar to the NAO model that is used throughout the paper. Section 4 details the analytical solution proposed to calculate the coupled joint angle exactly. Simulation results are presented in section 5 followed by the discussion section.

\section{Modeling Convention}

Khalil and Kleinfinger [10] proposed a modified convention, named $D H K K$, for geometric modeling from the Denavit-Hartenberg convention [11]. There are four $D H K K$ parameters required to go from coordinate frame $F_{i-1}$ to $F_{i}$, one for each transformation. Parameters are denoted by $a_{i}, \alpha_{i}, d_{i}$ and $\theta_{i}^{*} . a_{i}$ and $d_{i}$ are distances, $\alpha_{i}$ and $\theta_{i}^{*}$ are angles. Figure 2 shows the four parameters at stage $i$. They involve the three axes $\boldsymbol{z}_{\boldsymbol{i}-\mathbf{1}}, \boldsymbol{z}_{\boldsymbol{i}}$, and $\boldsymbol{z}_{\boldsymbol{i}+\mathbf{1}}$.

- $a_{i}$ is the algebraic distance $P_{i-1} P_{i-1}^{\prime}$ along $\boldsymbol{x}_{\boldsymbol{i - 1}}$.

$-\alpha_{i}$ is the rotation angle about $\boldsymbol{x}_{\boldsymbol{i - 1}}$ between $\boldsymbol{z}_{\boldsymbol{i}-\boldsymbol{1}}$ and $\boldsymbol{z}_{\boldsymbol{i}}$.

$-d_{i}$ is the algebraic distance $P_{i-1}^{\prime} P_{i}$ along $\boldsymbol{z}_{\boldsymbol{i}}$ axis.

- $\theta_{i}^{*}$ is the rotation angle about $\boldsymbol{z}_{\boldsymbol{i}}$ between $\boldsymbol{x}_{\boldsymbol{i}-\boldsymbol{1}}$ and $\boldsymbol{x}_{\boldsymbol{i}}$.

where $\boldsymbol{x}_{\boldsymbol{i}-1}$ is along the segment that is orthogonal to $\boldsymbol{z}_{\boldsymbol{i}-\boldsymbol{1}}$ and $\boldsymbol{z}_{\boldsymbol{i}}$ axes, from $\boldsymbol{z}_{\boldsymbol{i - 1}}$ to $\boldsymbol{z}_{\boldsymbol{i}} \cdot \boldsymbol{x}_{\boldsymbol{i}}$ is along the segment that is orthogonal to $\boldsymbol{z}_{\boldsymbol{i}}$ and $\boldsymbol{z}_{\boldsymbol{i}+\boldsymbol{1}}$ axes, from 


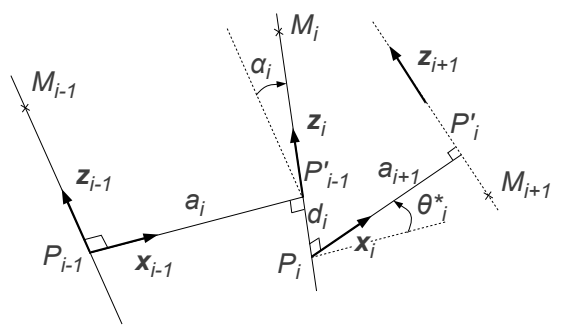

Fig. 2: Definition of $D H K K$ parameters for geometric modeling.

$\boldsymbol{z}_{\boldsymbol{i}}$ to $\boldsymbol{z}_{\boldsymbol{i}+\mathbf{1}} . P_{i}$ is the intersection point of $\boldsymbol{x}_{\boldsymbol{i}}$ with $\boldsymbol{z}_{\boldsymbol{i}} . P_{i}^{\prime}$ is the intersection point of $\boldsymbol{x}_{\boldsymbol{i}}$ with $\boldsymbol{z}_{\boldsymbol{i}+\mathbf{1}} \cdot M_{i}$ is a point that belongs to $\boldsymbol{z}_{\boldsymbol{i}}$ axis.

The coordinate frame transformation $T_{i}$ from $F_{i}$ to $F_{i-1}$, with $F_{i}=\left(P_{i}, \boldsymbol{x}_{\boldsymbol{i}}, \boldsymbol{y}_{\boldsymbol{i}}, \boldsymbol{z}_{\boldsymbol{i}}\right)$, is written as follows, where $R$ stands for rotation and $D$ for translation:

$$
\begin{aligned}
T_{i} & =D_{\boldsymbol{x}_{\boldsymbol{i}-\mathbf{1}}\left(a_{i}\right) R_{\boldsymbol{x}_{\boldsymbol{i}-\mathbf{1}}}\left(\alpha_{i}\right) D_{\boldsymbol{z}_{\boldsymbol{i}}}\left(d_{i}\right) R_{\boldsymbol{z}_{\boldsymbol{i}}}\left(\theta_{i}^{*}\right)} \\
T_{i} & =\left(\begin{array}{cccc}
\cos \theta_{i}^{*} & -\sin \theta_{i}^{*} & 0 & a_{i} \\
\cos \alpha_{i} \sin \theta_{i}^{*} & \cos \alpha_{i} \cos \theta_{i}^{*} & -\sin \alpha_{i} & -d_{i} \sin \alpha_{i} \\
\sin \alpha_{i} \sin \theta_{i}^{*} & \sin \alpha_{i} \cos \theta_{i}^{*} & \cos \alpha_{i} & d_{i} \cos \alpha_{i} \\
0 & 0 & 0 & 1
\end{array}\right)
\end{aligned}
$$

In the following sections we will use the reduced notation: $T_{x}=\left(\begin{array}{cc}R_{x} & D_{x} \\ 0 & 1\end{array}\right)$, where $R_{x}$ represents the rotation matrix, and $D_{x}$ the translation matrix to be applied.

\section{Model of Legs}

Figure 3 shows the skeleton of the humanoid trunk and legs in the reference position where legs are stretched vertically. The first joints at the hips are coupled, and their axes are inclined. The right-hand side of the figure shows the values of lengths and offsets related to two versions of NAO legs, of the 3D Soccer Simulation League (3D-SSL) and of the Standard Platform League (SPL). The inputs, namely points $M_{i}$ and axes $\boldsymbol{z}_{i}$ in the reference position of figure 3 , that are needed to calculate the $D H K K$ parameters automatically $[12,13]$ are also given. Table 1 presents the different values of the $D H K K$ parameters that are related to the $\mathrm{NAO} \operatorname{leg}(\xi=1$ for the right leg, and $\xi=-1$ for the left leg). 


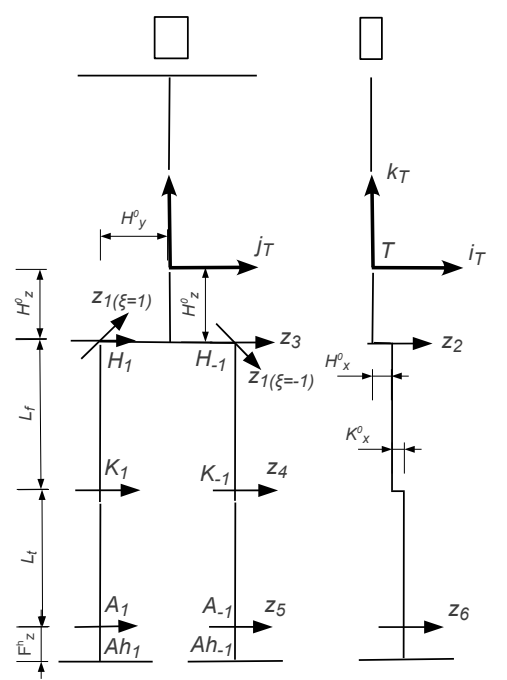

\begin{tabular}{llc}
\hline Offset/Length & \multicolumn{2}{l}{ 3DSSL leg SPL leg } \\
\hline Hip Offset Z: $H_{z}^{o}$ & 0.115 & 0.085 \\
Hip Offset Y: $H_{y}^{o}$ & 0.055 & 0.050 \\
Hip Offset X: $H_{x}^{o}$ & 0.010 & 0.000 \\
Knee Offset X: $K_{x}^{o}$ & 0.005 & 0.000 \\
Femur length: $L_{f}$ & 0.120 & 0.100 \\
Tibia length: $L_{t}$ & 0.100 & 0.103 \\
Foot Height: $F_{z}^{h}$ & 0.050 & 0.045 \\
\hline
\end{tabular}

\begin{tabular}{lr}
\hline Point $M_{i}$ & Joint axis $\boldsymbol{z}_{\boldsymbol{i}}$ \\
\hline$M_{s 1}=T$ & $\boldsymbol{z}_{\boldsymbol{s} \mathbf{1}}=\boldsymbol{k}_{\boldsymbol{T}}=[0,0,1]^{T}$ \\
$M_{s 2}=T$ & $\boldsymbol{z}_{\boldsymbol{s} \mathbf{2}}=\boldsymbol{k}_{\boldsymbol{T}}=[0,0,1]^{T}$ \\
$M_{1}=H$ & $\boldsymbol{z}_{\mathbf{1}}=[0, \cos (\pi / 4), \xi \sin (\pi / 4)]^{T}$ \\
$M_{2}=H$ & $\boldsymbol{z}_{\mathbf{2}}=\boldsymbol{i}_{\boldsymbol{T}}=[1,0,0]^{T}$ \\
$M_{3}=H$ & $\boldsymbol{z}_{\mathbf{3}}=\boldsymbol{j}_{\boldsymbol{T}}=[0,1,0]^{T}$ \\
$M_{4}=K$ & $\boldsymbol{z}_{\mathbf{4}}=\boldsymbol{j}_{\boldsymbol{T}}=[0,1,0]^{T}$ \\
$M_{5}=A$ & $\boldsymbol{z}_{\mathbf{5}}=\boldsymbol{j}_{\boldsymbol{T}}=[0,1,0]^{T}$ \\
$M_{6}=A$ & $\boldsymbol{z}_{\mathbf{6}}=\boldsymbol{i}_{\boldsymbol{T}}=[1,0,0]^{T}$ \\
$M_{e 1}=A h$ & $\boldsymbol{z}_{\boldsymbol{e} \mathbf{1}}=\boldsymbol{k}_{\boldsymbol{T}}=[0,0,1]^{T}$ \\
$M_{e 2}=A h$ & $\boldsymbol{z}_{\boldsymbol{e} \mathbf{2}}=-\boldsymbol{j}_{\boldsymbol{T}}=[0,-1,0]^{T}$ \\
\hline
\end{tabular}

Fig. 3: Humanoid skeleton with notations. $M_{e 2}=A h \quad \boldsymbol{z}_{\boldsymbol{e} \mathbf{2}}=-\boldsymbol{j}_{\boldsymbol{T}}=[0,-1,0]^{T}$ Frontal view on the left-hand side and sagittal view on the right-hand side.

Table 1: Leg's DHKK parameters. ${ }^{(\mathrm{a})} r=\left(H_{x}^{o}-K_{x}^{o}\right) / L_{f}$. $^{(\mathrm{b})} \delta=\tan ^{-1}(r)$.

\begin{tabular}{lccccc}
\hline $\mathrm{i}$ & $a_{i}[\mathrm{~m}]$ & $\alpha_{i}[\mathrm{rad}]$ & $d_{i}[\mathrm{~m}]$ & $\theta_{i}^{*}[\mathrm{rad}]$ & joint \\
\hline $\mathrm{s}$ & 0 & 0 & $-\left(H_{z}^{o}-H_{y}^{o}\right)$ & $\pi$ & dummy \\
1 & $H_{x}^{o}$ & $\pi / 4(2-\xi)$ & $-\xi \cdot \sqrt{2} \cdot H_{y}^{o}$ & $\theta_{1}-\pi / 2$ & hip yaw-pitch \\
2 & 0 & $\pi / 2$ & 0 & $\theta_{2^{+}}=\theta_{2}+\xi .3 \pi / 4$ & hip roll \\
3 & 0 & $\pi / 2$ & 0 & $\theta_{3}+\pi-\delta(\mathrm{b})$ & hip pitch \\
4 & $L_{f} \sqrt{1+r^{2}}(\mathrm{a})$ & 0 & 0 & $\theta_{4}+\delta$ & knee pitch \\
5 & $L_{t}$ & 0 & 0 & $\theta_{5}$ & ankle pitch \\
6 & 0 & $\pi / 2$ & 0 & $\theta_{6}-\pi / 2$ & ankle roll \\
$\mathrm{e}$ & 0 & $\pi / 2$ & $-F_{z}^{h}$ & $\pi / 2$ & dummy \\
\hline
\end{tabular}

The geometric model for the NAO humanoid leg can be written as:

$$
T_{0}=T_{s} T_{1} T_{2} T_{3} T_{4} T_{5} T_{6} T_{e}
$$

where

$-T_{s}$ involves the first three $z$ axes,

$-T_{e}$ involves the last three $z$ axes,

- $T_{i}$ involves joint rotation of angle $\theta_{i}^{*}$, and axes $\boldsymbol{z}_{\boldsymbol{i - 1}}, \boldsymbol{z}_{\boldsymbol{i}}$, and $\boldsymbol{z}_{\boldsymbol{i}+\mathbf{1}}$,

- $T_{0}$ is the homogeneous matrix given by the user, that represents the wanted orientation $R_{0}$ of the foot's sole in the TCF, and the position $D_{0}$ of the 
projection of the ankle on the sole, named $A_{h}$, in the TCF. $R_{0}$ is the matrix whose columns contain the coordinates of the Sole Coordinate Frame (SCF) axes expressed in the TCF. $R_{0}$ can be interpreted as the transformation matrix to pass from the SCF into the TCF.

\section{Analytical Solution}

\subsection{Objective}

We assume that feet always remain parallel to the horizontal ground. This means that there is a rotation about the vertical of a footprint with respect to the other. The angle of this rotation is noted $\varphi_{s}$. The objective is to calculate the yaw angle

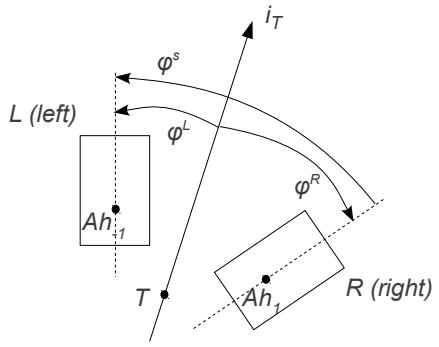

Fig. 4: Footprints in top view with right and left yaw angles $\varphi^{R}$ and $\varphi^{L}$, and step angle $\varphi^{s}$.

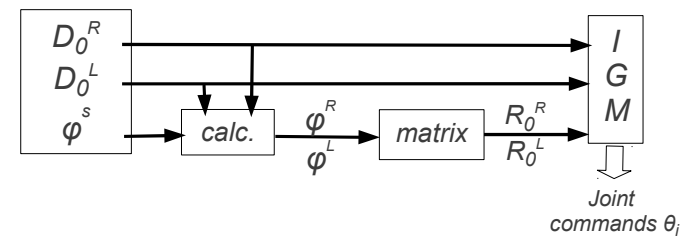

Fig. 5: Scheme of the analytical solution. IGM stands for Inverse Geometric Model.

$\varphi^{R}$ between the robot's trunk longitudinal axis $\boldsymbol{i}_{\boldsymbol{T}}$ and the right foot axis. The yaw angle with the left foot is $\varphi^{L}=\varphi^{R}+\varphi^{s}$ (Fig. 4).

The inputs for the analytical solution are the following:

- the 3D position of the right foot ankle projection within the TCF, $D_{0}^{R}$,

- the 3D position of the left foot ankle projection within the TCF, $D_{0}^{L}$,

- the angle between the right foot and the left foot, $\varphi^{s}$.

Figure 5 shows the scheme of the analytical solution that allows calculating all joint angles exactly taking into account the coupling between yaw-pitch joints at the hips. The next section details the calc. box of the scheme.

\subsection{Analytical expression of tangent of hip yaw-pitch joint angle, $\theta_{1}$}

Equation 3 leads to ${ }^{3}$ :

$$
\begin{aligned}
R_{123}\left(D_{4}+R_{4} D_{5}\right) & =D^{\prime} \\
R_{123} R_{456} & =R^{\prime}
\end{aligned}
$$

$\overline{3}^{3} R_{x y}=R_{x} R_{y}$ 
with

$$
\begin{aligned}
& R^{\prime}=R_{s}^{T} R_{0} R_{e}^{T} \\
& D^{\prime}=-D_{1}+R_{s}^{T}\left(D_{0}-D s-R_{0} R_{e}^{T} D_{e}\right)
\end{aligned}
$$

Squaring the first equation gives $\left(D_{4}+R_{4} D_{5}\right)^{2}=D^{\prime 2}$ that allows to solve for the knee angle $\theta_{4}$.

By replacing $R_{123}$ from Eq. (5) into Eq. (4), we get the following system:

$$
\begin{aligned}
& R_{6} \boldsymbol{u}=R_{5}^{T} \boldsymbol{v} \\
& R_{123}=R^{\prime \prime}
\end{aligned}
$$

with

$$
\begin{aligned}
\boldsymbol{u} & =R^{\prime T} D^{\prime} \\
\boldsymbol{v} & =D_{5}+R_{4}^{T} D_{4} \\
R^{\prime \prime} & =R^{\prime} R_{6}^{T} R_{5}^{T} R_{4}^{T}
\end{aligned}
$$

$\boldsymbol{u}$ can also be expressed as:

$$
\boldsymbol{u}=-D_{e}+R_{e} R_{0}^{T} R_{s}\left[-D_{1}+R_{s}^{T}\left(D_{0}-D_{s}\right)\right]
$$

By taking the last column of both matrices in Eq. (9), it comes ${ }^{4}$ :

$$
\begin{aligned}
R_{123} \cdot C_{3} & =R^{\prime} R_{6}^{T}[0,0,1]^{T} \\
{\left[\begin{array}{c}
s_{1} s_{2^{+}} \\
\frac{\sqrt{2}}{2}\left(-\xi c_{1} s_{2+}+c_{2+}\right) \\
\frac{\sqrt{2}}{2}\left(-c_{1} s_{2+}-\xi c_{2+}\right)
\end{array}\right] } & =R^{\prime}\left[\begin{array}{c}
-c_{6} \\
s_{6} \\
0
\end{array}\right]
\end{aligned}
$$

$\theta_{2}$ varies inside $[-\pi / 2, \epsilon]$ for the right leg and inside $[-\epsilon, \pi / 2]$ for the left leg, where $\epsilon$ represents a positive value that is less than $\pi / 4$. Hence $\theta_{2+}$ (see Tab. 1) varies inside $\left[\frac{\pi}{4}, \epsilon+\frac{3 \pi}{4}\right]$ for the right leg and inside $\left[-\epsilon-\frac{3 \pi}{4},-\frac{\pi}{4}\right]$ for the left leg. Therefore $s_{2+}$ is never equal to zero and we get ${ }^{5}$ :

$$
t_{1}=\tan \theta_{1}=-\frac{1}{\sqrt{2}} \frac{-R_{11}^{\prime} c_{6}+R_{12}^{\prime} s_{6}}{-\left(\xi R_{21}^{\prime}+R_{31}^{\prime}\right) c_{6}+\left(\xi R_{22}^{\prime}+R_{32}^{\prime}\right) s_{6}}
$$

To determine $\theta_{6}$ we use the last line of matrix equation (8):

$$
-c_{6} u_{x}+s_{6} u_{y}=0
$$

which gives $c_{6}= \pm u_{y} /\left(u_{x}^{2}+u_{y}^{2}\right)$ and $s_{6}= \pm u_{x} /\left(u_{x}^{2}+u_{y}^{2}\right)$. Therefore:

$$
t_{1}=\frac{1}{\sqrt{2}} \frac{R_{11}^{\prime} u_{y}-R_{12}^{\prime} u_{x}}{-\left(\xi R_{21}^{\prime}+R_{31}^{\prime}\right) u_{y}+\left(\xi R_{22}^{\prime}+R_{32}^{\prime}\right) u_{x}}
$$

\footnotetext{
${ }^{4} s_{x}$ and $c_{x}$ stand respectively for $\sin \theta_{x}$ and $\cos \theta_{x}$

${ }^{5} R_{n_{1} n_{2}}$ stands for the element at row $n_{1}$ and column $n_{2}$ of matrix $R$
} 
with:

$$
\begin{aligned}
\boldsymbol{u} & =-D_{e}+R^{\prime T} \overline{D_{0}} \\
\overline{D_{0}} & =-D_{1}+R_{s}^{T}\left(D_{0}-D_{s}\right) \\
u_{x} & =R_{11}^{\prime} x_{\overline{D_{0}}}+R_{21}^{\prime} y_{\overline{D_{0}}}+R_{31}^{\prime} z_{\overline{D_{0}}} \\
u_{y} & =-F_{z}^{h}+R_{12}^{\prime} x_{\overline{D_{0}}}+R_{22}^{\prime} y_{\overline{D_{0}}}+R_{32}^{\prime} z_{\overline{D_{0}}}
\end{aligned}
$$

Then,

$$
t_{1}=\frac{\left(-F_{z}^{h} R_{11}^{\prime}+y_{\overline{D_{0}}} d_{1}+z_{\overline{D_{0}}} d_{3}\right) / \sqrt{2}}{\left(\xi R_{21}^{\prime}+R_{31}^{\prime}\right) F_{z}^{h}+\left(\xi d_{1}+d_{3}\right) x_{\overline{D_{0}}}+d_{2}\left(y_{\overline{D_{0}}}-\xi z_{\overline{D_{0}}}\right)}
$$

with:

$$
d_{1}=R_{11}^{\prime} R_{22}^{\prime}-R_{12}^{\prime} R_{21}^{\prime}, \quad d_{2}=R_{21}^{\prime} R_{32}^{\prime}-R_{22}^{\prime} R_{31}^{\prime}, \quad d_{3}=R_{11}^{\prime} R_{32}^{\prime}-R_{12}^{\prime} R_{31}^{\prime}
$$

\subsection{Determination of the body yaw angle}

Because of the coupling of both yaw-pitch joints at the hips, the following constraint of equality of right and left joint angles must be satisfied:

$$
\tan \theta_{1}^{R}=\tan \theta_{1}^{L}
$$

Given that the foot soles remain horizontal and that the trunk can be pitched forward or backward by an angle $\eta$ in the sagittal plane during the walk, $R_{0}$ can be written as:

$$
R_{0}=\left[\begin{array}{ccc}
c \eta & 0 & -s \eta \\
0 & 1 & 0 \\
s \eta & 0 & c \eta
\end{array}\right]\left[\begin{array}{ccc}
c & -s & 0 \\
s & c & 0 \\
0 & 0 & 1
\end{array}\right]
$$

which is the matrix product of the rotation matrix of angle $(-\eta)$ about the $y$ axis and the rotation matrix of the walking step, with $c=\cos \varphi$ and $s=\sin \varphi$. $\varphi=\varphi^{R}$ for the right leg, and $\varphi=\varphi^{L}$ for the left leg.

Therefore $R^{\prime}$ becomes:

$$
\begin{gathered}
R^{\prime}=\left[\begin{array}{ccc}
-s c \eta-s \eta & -c c \eta \\
c & 0 & -s \\
s s \eta & -c \eta & c s \eta
\end{array}\right] \\
d_{1}=c s \eta, \quad d_{2}=-c c \eta, \quad d_{3}=s
\end{gathered}
$$

and Eqs (17) and (18) become:

$$
\begin{aligned}
t_{1} & =\frac{1}{\sqrt{2}} \frac{p_{1} \tan \varphi+p_{2}}{p_{3} \tan \varphi+p_{4}} \\
\frac{p_{1}^{R} \tan \varphi^{R}+p_{2}^{R}}{p_{3}^{R} \tan \varphi^{R}+p_{4}^{R}} & =\frac{p_{1}^{L} \tan \varphi^{L}+p_{2}^{L}}{p_{3}^{L} \tan \varphi^{L}+p_{4}^{L}}
\end{aligned}
$$


Replacing $\varphi^{L}$ by $\varphi^{R}+\varphi^{s}$, and noting $t=\tan \varphi^{R}, t^{s}=\tan \varphi^{s}$ leads to the 2 nd order equation:

$$
t^{2}+B \cdot t+C=0
$$

with

$$
\begin{aligned}
B & =\left(p_{1}^{R} \sigma_{1}+p_{2}^{R} \sigma_{2}-p_{3}^{R} \sigma_{3}-p_{4}^{R} \sigma_{4}\right) / A=B^{\prime} / A \\
C & =\left(p_{2}^{R} \sigma_{1}-p_{4}^{R} \sigma_{3}\right) / A=C^{\prime} / A \\
A & =p_{1}^{R} \sigma_{2}-p_{3}^{R} \sigma_{4} \\
p_{1}^{R, L} & =F_{z}^{h} c \eta+z \overline{D_{O}^{R, L}}, p_{2}^{R, L}=y \overline{D_{O}^{R, L}} s \eta \\
p_{3}^{R, L} & =F_{z}^{h} s \eta+x \overline{D_{O}^{R, L}}, p_{4}^{R, L}=\xi\left(F_{z}^{h}+c \eta z \overline{D_{O}^{R, L}}+s \eta x \overline{D_{O}^{R, L}}\right)-c \eta y \overline{D_{O}^{R, L}} \\
\sigma_{1} & =p_{3}^{L} t^{s}+p_{4}^{L}, \sigma_{2}=p_{3}^{L}-p_{4}^{L} t^{s}, \sigma_{3}=p_{1}^{L} t^{s}+p_{2}^{L}, \sigma_{4}=p_{1}^{L}-p_{2}^{L} t^{s}
\end{aligned}
$$

The solution for $\varphi^{R}$ is the one with the lowest magnitude:

$$
\Rightarrow t=\frac{1}{2}\left(-B+\operatorname{sign}(B) \cdot \sqrt{B^{2}-4 C}\right)
$$

Then

$$
\varphi^{R}=\tan ^{-1}(t)
$$

The other one is the solution where the feet are pointing inwards with an approximately $90[\mathrm{deg}]$ angle with respect to the first solution. If $\left(A, B^{\prime}\right)=(0,0)$ there is no solution. If $B^{\prime}=0, t=-\sqrt{-C}$. If $A=0$ then $t=-C^{\prime} / B^{\prime}$.

\section{$5 \quad$ Simulation Results}

The analytical solution was embedded in the locomotion code of NAO to be used in the 3D-SSL software (SimSpark application for the RoboCup 3D-SSL [14,15], namely rcssserver3d, based on the Open Dynamics Engine (ODE)). This code enables to calculate the joint command angles in real time to be sent to the server to make the NAO client walk on the simulator. Walking patterns were designed using the model of the $3 \mathrm{D}$ linear inverted pendulum and the Zero Moment Point technique [9].

The analytical solution was tested for three walking patterns. The first one is a forward walk of $0.1[\mathrm{~m}]$, the second one a sideways walk of $0.1[\mathrm{~m}]$, and the third one a turn-in-place of $60[\mathrm{deg}]$. In the forward walk the left leg makes a $0.05[\mathrm{~m}]$ step forward, then the right leg executes a $0.1[\mathrm{~m}]$ step forward, and finally the left leg makes a $0.05[\mathrm{~m}]$ step to come parallel with the right leg. In the sideways walk, the left leg executes a left sidestep of $0.05[\mathrm{~m}]$, then the right leg makes a left sidestep to comes parallel with the left leg. This sequence is reproduced once again to cover a total distance of $0.1[\mathrm{~m}]$. In the turn-in-place walk the left leg executes a $30[\mathrm{deg}]$ left turn, then the right leg makes also a $30[\mathrm{deg}]$ left turn 

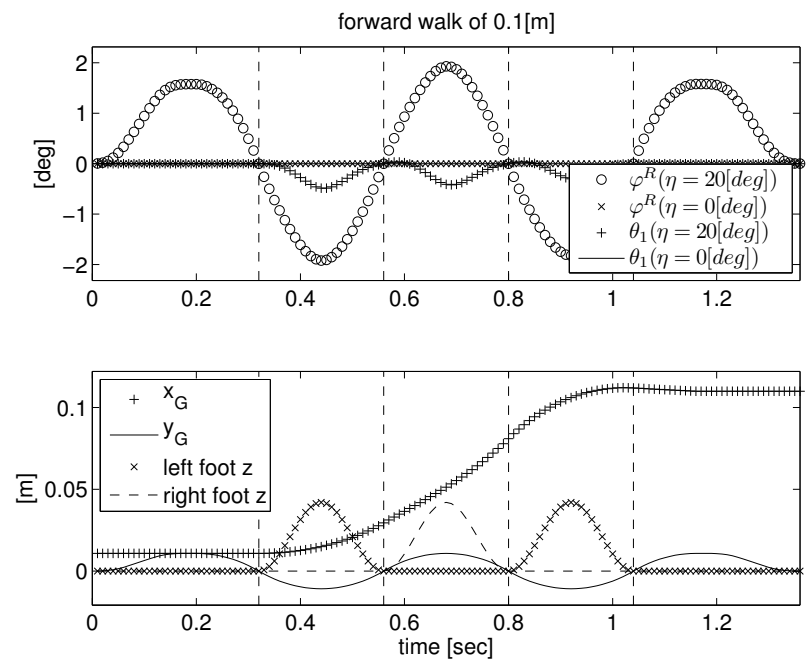

Fig. 6: Variations of the right yaw angle $\varphi^{R}$ and the coupled-yaw joint angle $\theta_{1}$ along a $0.1[\mathrm{~m}]$ forward walk. Angles values are given for the straight torso $(\eta=0[\mathrm{deg}])$, and for a forward inclination of $20[\mathrm{deg}]$ of the torso in the sagittal plane $(\eta=20[\mathrm{deg}])$.
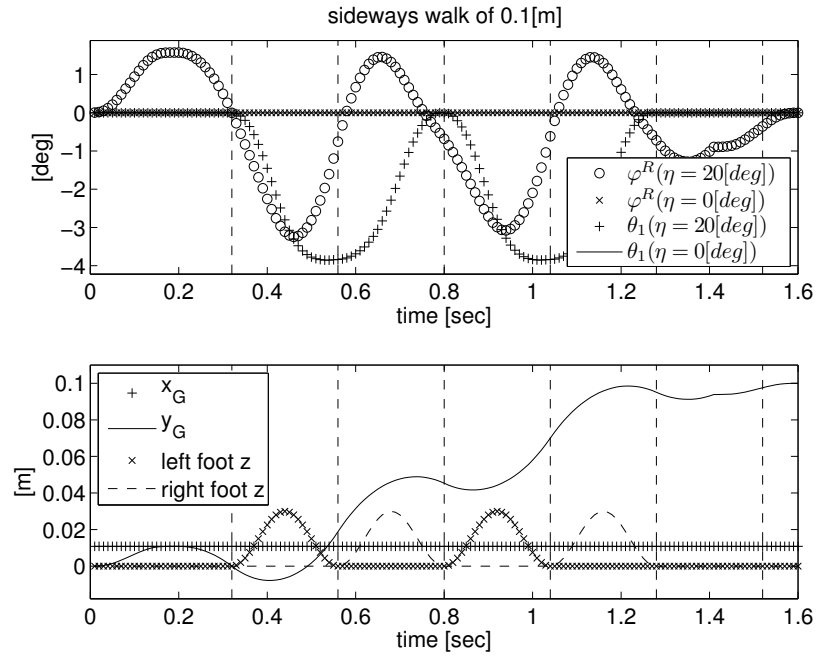

Fig. 7: Variations of the right yaw angle $\varphi^{R}$ and the coupled-yaw joint angle $\theta_{1}$ along a sideways walk $0.1[\mathrm{~m}]$. Angles values are given for the straight torso $(\eta=0[\mathrm{deg}])$, and for a forward inclination of $20[\mathrm{deg}]$ of the torso in the sagittal plane $(\eta=20[\mathrm{deg}])$.

to get parallel with left leg. The sequence is reproduced once again to cover a total angle of $60[\mathrm{deg}]$. The center of rotation is located at $0.01[\mathrm{~m}]$ behind the middle of the ankles to give more space for the heels and prevent them from colliding into each other during the rotation motion of the leading leg. Each step 

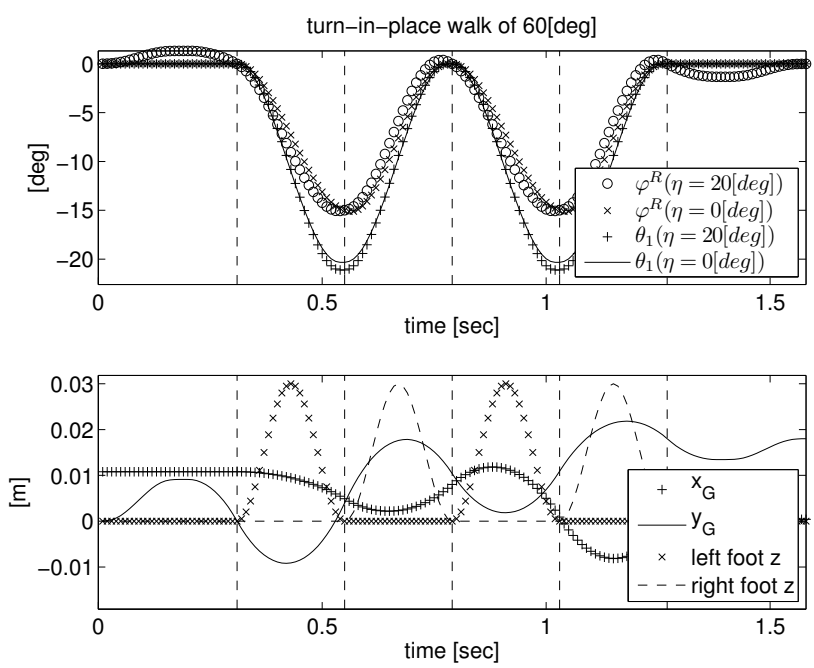

Fig. 8: Variations of the right yaw angle $\varphi^{R}$ and the coupled-yaw joint angle $\theta_{1}$ along a left turn-in-place of $60[\mathrm{deg}]$. Angles values are given for the straight torso $(\eta=0[\mathrm{deg}])$, and for a forward inclination of $20[\mathrm{deg}]$ of the torso in the sagittal plane $(\eta=20[\mathrm{deg}])$.

lasts $0.24[\mathrm{sec}]$. Before executing the first step of every waking pattern, the robot sways its hips outward, then inward to initiate the step. After the last step, there is also an outward-inward hip sway to stop the lateral oscillation of the torso. Figures 6,7 and 8 display the variations over time of the yaw angle $\varphi^{R}$ and of the coupled-yaw-joint angle $\theta_{1}$ for the three walking patterns respectively, with the torso in straight position $(\eta=0[\mathrm{deg}])$ and with the torso inclined forward $(\eta=20[\mathrm{deg}])$. The bottom part of each of the figures gives the variation over time of the horizontal coordinates of the center of mass $\left(x_{G}\right.$ along the longitudinal axis, $y_{G}$ along the lateral axis), and the variation with time of both foot heights.

In the case where the torso remains straight $(\eta=0[\mathrm{deg}])$, forward and sideways walking do not require the yaw coupled joint $\theta_{1}$ to participate in the leg motion. Actually this joint angle remains equal to zero. On the contrary the turn-in-place walk requires the use of the yaw coupled joint to design the turning trajectories of the legs.

During forward and sideways walking steps, the inclination of the torso requires the actuation of the yaw coupled-joint angle to maintain feet parallel. This leads to an oscillation of the torso about the yaw axis, of approx. $4[\mathrm{deg}]$ of peak to peak amplitude for a $20[\mathrm{deg}]$ inclination (see $\varphi^{R}$, angle between torso longitudinal axis and right leg longitudinal axis). In the forward walk the oscillation of the torso is symmetrical with respect to the longitudinal axis, whereas in the left sidestep the torso oscillates more right than left, and vice-versa. During the turn-in-place walk, the forward inclination of the torso leads to similar variations of the yaw coupled-joint angle, with some light fluctuations compared with the turn-in-place with straight torso. The torso longitudinal axis does not remain 
rigorously along the bisector of the angle between both feet, i.e. $\varphi^{L}$ is not equal to $\varphi^{R}$ except at the foot impacts.

\section{Discussion}

The analytical solving allows designing double support phases during the walk. For a fixed configuration of footprints the desired inputs to the inverse kinematics can be the center of mass of the robot and the torso bending angle in the sagittal plane, the roll angle being kept to $0[\mathrm{deg}]$. Due to the underactuation at the hips the yaw angle is constrained and can be calculated in real time to command the coupled joint angle. Compared with other approximate solutions [7-9], this analytical solving enables to calculate joint angles exactly and to prevent feet from sliding on the ground. In addition this contributes to reduce the stress on the joints.

The calculation presented here assumes that the feet soles are horizontal and that the torso remains vertical or is bent in the sagittal plane. This calculation could be especially useful for walking algorithms that embed closed loop control of torso inclination like the technique adopted by Gouaillier et al. [16] or the one used by Glaser et al. [17] for the NAO robot. However, if we take the same foot trajectory shape for the inclined torso as for the straight torso to design longitudinal and lateral walking patterns where feet remain parallel, the coupled yaw-pitch joint must also be controlled, this is because the roll axis at the hip is no more horizontal and therefore cannot move the foot in the frontal plane without actuating the coupled joint. This results in a yaw motion of the torso about the vertical, in the direction of the support leg when the other leg is lift off, and in the other direction when the other leg goes down for landing.

In the case of instantaneous double support phases, the swing trajectory of the leg can be tuned to ensure fixed torso heading by transferring the slight rotation of the torso to the swinging leg.

\section{Conclusion}

This paper has presented an analytical solution for the yaw coupled joint at the hips of the NAO humanoid robot. The calculation of the yaw angle can be made in real time, allowing the control of the coupled joint to enable the exact tracking of the foot Cartesian trajectories. From an inverse kinematics point of view the definition of the robot's center of mass position and the bending angle in the sagittal plane of the torso can be used as inputs to design walking moves. The roll joint is kept nul. The solution proposed is especially useful for the command of the joints in the case of turning steps, or in all cases of walking patterns when the torso is inclined in the sagittal plane. In the case of instantaneous double

support, it can be useful to design swing leg trajectories carefully in the trunk reference frame to avoid rotation of the torso about the vertical. 


\section{References}

1. Gouaillier, D., Hugel, V., Blazevic, P., Kilner, C., Monceaux, J., Lafourcade, P., Marnier, B., Serre, J. and Maisonnier, B., Mechatronic design of NAO humanoid. Int. Conf. on Robotics and Automation, 2009, 769-774.

2. Czarnetzki, S., Kerner, S., Urbann, O., Applying dynamic walking control for biped robots, Robot Soccer World Cup XIII. Lecture Notes in Computer Science, 2010, 5949:69-80.

3. Graf, C., Härtl, A., Röfer, T., Laue, T., A Robust Closed-Loop Gait for the Standard Platform League Humanoid, Proc. of the 4th Workshop on Humanoid Soccer Robots, 2009, 30-37.

4. Wenk, F. and Röfer, T., Online Generated Kick Motions for the NAO Balanced Using Inverse Dynamics, RoboCup Symp., 2013.

5. Alcaraz-Jiménez, J.J., Missura, M., and Behnke, S., Lateral disturbance rejection for the NAO robot, RoboCup 2012: Robot Soccer World Cup XVI. Lecture Notes in Computer Science, 2013, 7500:1-12.

6. Hengst, B. and Lange, M. and White, B., Learning ankle-tilt and foot-placement control for flat-footed bipedal balancing and walking, IEEE-RAS Int. Conf. on Humanoid Robots, 2011, 288-293.

7. Röfer, T., Laue, T., Müller, J., Bösche, O., Burchardt, A., Damrose, E., Gillmann, K., Graf, C., de Haas, T. J., Härtl, A., Rieskamp, A., Schreck, A., Sieverdingbeck, I. and Worch, J.-H., B-Human Team Report and Code Release 2009. http://www.bhuman.de/downloads/bhuman09_coderelease.pdf

8. Alcaraz-Jiménez, J.J., Robust Fee- dback Control of Omnidirectional Biped Gait on NAO Humanoid Robots, Ph.D. Thesis, University of Murcia, Spain, 2013.

9. Hugel, V. and Jouandeau, N., Walking patterns for real time path planning simulation of humanoids, IEEE RO-MAN, 2012, 424-430.

10. Khalil, W. and Kleinfinger, J-F., A new geometric notation for open and closedloop robots. IEEE Int. Conf. on Robotics and Automation, 1986, 1174-1180.

11. Denavit, J. and Hartenberg, R.S., A kinematic notation for lower-pair mechanisms based on matrices. Trans ASME J. Appl. Mech, 1955, 23:215-221.

12. Zorjan, M. and Hugel, V. and Blazevic, P., Influence of hip joint axes change of orientation on power distribution in humanoid motion, IEEE Int. Conf. on Automation, Robots and Applications, 2011, 271-276.

13. Zorjan, M. and Hugel, V., Generalized Humanoid Leg Inverse Kinematics to Deal With Singularities. IEEE Int. Conf. on Robotics and Automation, 2013, 4791-4796.

14. Obst, O. and Rollmann, M., Spark - A Generic Simulator for Physical Multi-Agent Simulations, Multiagent System Technologies, Lecture Notes in Computer Science, 2004, 3187:243-257.

15. SimSpark, a generic physical multiagent simulator system for agents in threedimensional environments. http://simspark.sourceforge.net/.

16. Gouaillier, D. and Collette, C. and Kilner, C., Omni-directional closed-loop walk for NAO, IEEE-RAS Int. Conf. on Humanoid Robots, 2010, 448-454.

17. Glaser, S. and Dorer, K., Trunk Controlled Motion Framework, Proc. of the 8th Workshop on Humanoid Soccer Robots, 2013. 